UDC: $547.789 .1+547.722$

\title{
Synthesis and antimicrobial activity of 5-R-benzyl-2-(arylidenehydrazono)thiazolidin-4-ones
}

\author{
I. V. Drapak ${ }^{1}$, L. S. Logoyda ${ }^{2}$, N. Ye. Shtoyko ${ }^{1}$, M. I. Sulyma ${ }^{1}$, \\ T. I. Chaban ${ }^{1}$, V. S. Matiychuk ${ }^{3}$ \\ ${ }^{1}$ Danylo Halytsky Lviv National Medical University \\ 69, Pekarska Str., Lviv, Ukraine, 79010 \\ 2 I. Horbachevsky Ternopil National Medical University \\ m.Voli, 1, Ternopil, Ukraine, 46001 \\ ${ }^{3}$ Ivan Franko National University of Lviv \\ 1, Universytetska Str., Lviv, Ukraine, 79000 \\ iradrapak@ukr.net
}

\begin{abstract}
Aim. Study of the synthesis and antimicrobial activity of a series of 5-R-benzyl-2(arylidenehydrazono)thiazolidin-4-ones. Methods. Organic synthesis, analytical and spectral methods, assay of antimicrobial activity. Results. 5-R-benzyl-2-(arylidenehydrazono)thiazolidin-4-ones 6a-k and 7a,b have been synthesized in good yields via reaction of 2-bromo3 -arylpropanoates $3 a-j$ with thiosemicarbazones aromatic aldehydes and furfuraldehyde. Their structures of synthesized compounds were confirmed by ${ }^{1} \mathrm{H}$ NMR spectroscopy and elemental analysis. The synthesized compounds have been evaluated for antimicrobial activity against five bacterial strains (Escherichia coli, Klebsiella pneumoniae, Acinetobacter baumannii, Pseudomonas aeruginosa, Staphylococcus aureus) and two fungal strains (Candida albicans and Cryptococcus neoformans). According to the antimicrobial activity results, the tested compounds, $6 \mathrm{~b}, 6 \mathrm{~h}-\mathrm{k}$ and $7 \mathrm{a}$ were found to be active against Staphylococcus aureus whereas 6a, 6d, 6f, 6g, 7b - towards Cryptococcus neoformans. The relationship between the nature of substituents in aromatic moieties and [the] activity level was established. Conclusions. A series of new 5-R-benzyl-2-(arylidenehydrazono)thiazolidin-4-ones were synthesized. The compounds with antimicrobal activity have been found.
\end{abstract}

Keyword s: organic synthesis, arylation, 5-R-benzyl-2-(arylidenehydrazono)thiazolidin4-ones, antimicrobal activity.

(C) 2020 I. V. Drapak et al.; Published by the Institute of Molecular Biology and Genetics, NAS of Ukraine on behalf of Biopolymers and Cell. This is an Open Access article distributed under the terms of the Creative Commons Attribution License (http://creativecommons.org/licenses/by/4.0/), which permits unrestricted reuse, distribution, and reproduction in any medium, provided the original work is properly cited 


\section{Introduction}

The treatment of infectious diseases still remains an important and challenging problem because of a combination of factors including emerging infectious diseases and the increasing number of multi-drug resistant microbial pathogens. The therapeutic problem is of particular importance for hospitalised patients, immune suppressed patients with AIDS or undergoing anticancer therapy and organ transplantation. Though a large number of antibiotics and chemotherapeutics are available for medical use, the emergence of old and new antibiotic resistance caused in the last decades a substantial need for new classes of antibacterial agents. A potential approach to overcome the problem of resistance is to design innovative agents with a different mode of action so that no cross-resistance with the present drugs can occur.

Because heterocycles have a central position in both medicinal and organic chemistry, considerable attention has been focused on their syntheses. The nitrogen heterocycles in particular exhibit diverse biological and pharmacological activities partly due to the similarities with many natural and synthetic molecules with known biological activity. The substituted 4-thiazolidinones are one of the most extensively studied classes of heterocyclic compounds receiving much attention from synthetic organic as well as medicinal chemists because of the diverse range of their biological activities and obvious therapeutic properties [1-3]. They are used as antitubercular, antimicrobial, anti-inflammatory, antiviral and antiHIV agents [1-4]. In view of biological importance of 4-thiazolidinone derivatives we report the synthesis and primary evaluation of antimicrobial activity [of] new 2-(arylidenehydrazono)-thiazolidin-4-one. Among such compounds, 5-arylidene-2-(aryl2-ylmethylenehydrazono)thiazolidin-4-ones were studied. Their antimicrobial [5-9], antiviral [10], anti-inflammatory [11], antitumor $[12,13]$ [properties] have been reported. These compounds are also the inhibitors of various enzymes [14-16]. At the same time, the less conformational restrictions of 5-benzyl-4-thiazolidinone derivatives are practically unexplored.

\section{Materials and Methods}

Chemistry. All starting materials were purchased from Merck and used without purification. NMR spectra were determined with Varian Mercury 400 (400 MHz) spectrometer, in DMSO- $d_{6}$. Melting points were determined in open capillary tubes and are uncorrected. The purity of the compounds was checked by thin-layer chromatography performed with Merck Silica Gel 60 F254 aluminum sheets.

General procedure for preparation of 5-R-benzyl-2-(arylidenehydrazono)thiazolidin-4-ones (6a-k) and $(7 a, b)$. A mixture of 0.01 mole of thiosemicarbazone $\mathbf{4 a - d}$ or $\mathbf{5}$, 0.01 mole of ester $\mathbf{3 a - j}$ and $0.82 \mathrm{~g}$ ( 0.01 mole) of sodium acetate in $25 \mathrm{ml}$ of ethanol was refluxed for $4 \mathrm{~h}$. After cooling the precipitate was filtered off and recrystallized from ethanol.

5-Benzyl-2-I(2-methoxybenzylidene)hydrazonol-thiazolidin-4-on (6a). Yield $75 \%$, m.p. $229-230{ }^{\circ} \mathrm{C} .{ }^{1} \mathrm{H}$ NMR (400 MHz, [D6] 
DMSO): $\delta=12.02(\mathrm{~s}, 1 \mathrm{H}, \mathrm{NH}), 8.57(\mathrm{~s}, 1 \mathrm{H}$, $\mathrm{CH}=), 7.76\left(\mathrm{~d}, \mathrm{~J}=7.3 \mathrm{~Hz}, 1 \mathrm{H}, \mathrm{C}_{6} \mathrm{H}_{4}\right), 7.43(\mathrm{t}$, $\left.\mathrm{J}=7.9 \mathrm{~Hz}, 1 \mathrm{H}, \mathrm{C}_{6} \mathrm{H}_{4}\right), 7.36-7.22(\mathrm{~m}, 5 \mathrm{H}, \mathrm{Ph})$, $7.09\left(\mathrm{~d}, \mathrm{~J}=8.4 \mathrm{~Hz}, 1 \mathrm{H}, \mathrm{C}_{6} \mathrm{H}_{4}\right), 6.98(\mathrm{t}, \mathrm{J}=$ $\left.7.5 \mathrm{~Hz}, 1 \mathrm{H}, \mathrm{C}_{6} \mathrm{H}_{4}\right), 4.63(\mathrm{dd}, \mathrm{J}=9.8,4.1 \mathrm{~Hz}$, $1 \mathrm{H}, \mathrm{CH}), 3.83\left(\mathrm{~s}, 3 \mathrm{H}, \mathrm{CH}_{3} \mathrm{O}\right), 3.40(\mathrm{dd}, \mathrm{J}=$ 14.1, 4.1 Hz, $\left.1 \mathrm{H}, \mathrm{CH}_{2}\right), 3.01(\mathrm{dd}, \mathrm{J}=14.1$, $10.0 \mathrm{~Hz}, 1 \mathrm{H}, \mathrm{CH}_{2}$ ). Anal. Calcd. for $\mathrm{C}_{18} \mathrm{H}_{17} \mathrm{~N}_{3} \mathrm{O}_{2} \mathrm{~S}$ : C, 63.70; H, 5.05; N, 12.38 . Found: C, 64.02; H, 5.11; N, 12.45.

2 - (Benzylidenehydrazono) - 5 - (2 methylbenzyl)-thiazolidin-4-one (6b). Yield $72 \%$, m.p. $221-222{ }^{\circ} \mathrm{C} .{ }^{1} \mathrm{H}$ NMR (400 MHz, [D6]DMSO): $\delta=12.10(\mathrm{~s}, 1 \mathrm{H}, \mathrm{NH}), 8.40(\mathrm{~s}$, $1 \mathrm{H}, \mathrm{CH}=), 7.73(\mathrm{~d}, J=1.9 \mathrm{~Hz}, 1 \mathrm{H}), 7.71(\mathrm{~d}$, $J=3.7 \mathrm{~Hz}, 1 \mathrm{H}, \mathrm{Ar}), 7.47-7.38$ (m, 3H, Ar), 7.25-7.13 (m, 4H, Ar), 4.62 (dd, $J=11.1$, $3.8 \mathrm{~Hz}, 1 \mathrm{H}, \mathrm{CH}), 3.47$ (dd, $J=14.5,3.8 \mathrm{~Hz}$, $\left.1 \mathrm{H}, \mathrm{CH}_{2}\right), 2.96(\mathrm{dd}, J=14.5,11.1 \mathrm{~Hz}, 1 \mathrm{H}$, $\left.\mathrm{CH}_{2}\right), 2.31\left(\mathrm{~s}, 3 \mathrm{H}, \mathrm{CH}_{3}\right)$. Anal. Calcd. for $\mathrm{C}_{18} \mathrm{H}_{17} \mathrm{~N}_{3} \mathrm{OS}$ : C, 66.85; H, 5.30; N, 12.99 . Found: C, 66.48; H, 5.36; N, 12.84.

5 - ( 4 - Eth y l be n zyl) - 2 - I ( 4 methoxybenzylidene)-hydrazonol-thiazolidin4-one (6c). Yield $78 \%$, m.p. $222-223{ }^{\circ} \mathrm{C} .{ }^{1} \mathrm{H}$ NMR (400 MHz, [D6]DMSO): $\delta=11.94$ (s, $1 \mathrm{H}, \mathrm{NH}), 8.30(\mathrm{~s}, 1 \mathrm{H}, \mathrm{CH}=), 7.65(\mathrm{~d}, J=$ $\left.8.7 \mathrm{~Hz}, 2 \mathrm{H}, \mathrm{C}_{6} \mathrm{H}_{4} \mathrm{OCH}_{3}\right), 7.19(\mathrm{~d}, J=8.2 \mathrm{~Hz}$, $\left.2 \mathrm{H}, \mathrm{C}_{6} \mathrm{H}_{4} \mathrm{C}_{2} \mathrm{H}_{5}\right), 7.16(\mathrm{~d}, J=8.2 \mathrm{~Hz}, 2 \mathrm{H}$, $\left.\mathrm{C}_{6} \mathrm{H}_{4} \mathrm{C}_{2} \mathrm{H}_{5}\right), 6.99(\mathrm{~d}, J=8.7 \mathrm{~Hz}, 2 \mathrm{H}$, $\left.\mathrm{C}_{6} \mathrm{H}_{4} \mathrm{OCH}_{3}\right), 4.59(\mathrm{dd}, J=9.9,4.0 \mathrm{~Hz}, 1 \mathrm{H}$, $\mathrm{CH}), 3.35$ (dd, J = 14.5, 3.8 Hz, $\left.1 \mathrm{H}, \mathrm{CH}_{2}\right), 2.95$ $\left(\mathrm{dd}, J=14.1,10.0 \mathrm{~Hz}, 1 \mathrm{H}, \mathrm{CH}_{2}\right), 2.57$ (q, $J=$ $\left.7.6 \mathrm{~Hz}, 2 \mathrm{H}, \mathrm{CH}_{3} \mathrm{CH}_{2}\right), 1.16(\mathrm{t}, J=7.6 \mathrm{~Hz}, 3 \mathrm{H}$, $\mathrm{CH}_{3} \mathrm{CH}_{2}$ ). Anal. Calcd. for $\mathrm{C}_{20} \mathrm{H}_{21} \mathrm{~N}_{3} \mathrm{O}_{2} \mathrm{~S}: \mathrm{C}$, 65.37; H, 5.76; N, 11.43. Found: C, 65.50; H, $5.88 ; \mathrm{N}, 11.55$.

2 - (Benzylidenehydrazono) - 5-(4fluorobenzyl)-thiazolidin-4-one (6d). Yield
$81 \%$, m.p. $223-224{ }^{\circ} \mathrm{C} .{ }^{1} \mathrm{H}$ NMR (400 MHz, [D6]DMSO): $\delta=12.00(\mathrm{~s}, 1 \mathrm{H}, \mathrm{NH}), 8.32$ (s, $1 \mathrm{H}, \mathrm{CH}=), 7.72(\mathrm{dd}, \mathrm{J}=6.6,2.8 \mathrm{~Hz}, 2 \mathrm{H}, \mathrm{Ph})$, 7.47-7.41 (m, 3H, Ph), 7.33 (dd, $J=8.3$, $\left.5.8 \mathrm{~Hz}, 2 \mathrm{H}, \mathrm{C}_{6} \mathrm{H}_{4} \mathrm{~F}\right), 7.15(\mathrm{t}, J=8.8 \mathrm{~Hz}, 2 \mathrm{H}$, $\left.\mathrm{C}_{6} \mathrm{H}_{4} \mathrm{~F}\right), 4.63(\mathrm{dd}, J=9.4,4.3 \mathrm{~Hz}, 1 \mathrm{H}, \mathrm{CH})$, $3.37\left(\mathrm{dd}, J=14.2,4.2 \mathrm{~Hz}, 1 \mathrm{H}, \mathrm{CH}_{2}\right), 3.05$ (dd, $\left.J=14.1,9.5 \mathrm{~Hz}, 1 \mathrm{H}, \mathrm{CH}_{2}\right)$. Anal. Calcd. for $\mathrm{C}_{17} \mathrm{H}_{14} \mathrm{FN}_{3} \mathrm{OS}$ : C, 62.37; H, 4.31; N, 12.84 . Found: C, 62.09; H, 4.26; N, 12.88 .

5 - ( 4 - Fl и о r ob e nzyl) - 2 - I ( 2 methoxybenzylidene)-hydrazono/-thiazolidin4-one (6e). Yield $74 \%$, m.p. $230-231{ }^{\circ} \mathrm{C} .{ }^{1} \mathrm{H}$ NMR (400 MHz, [D6]DMSO): $\delta=11.98$ (s, $1 \mathrm{H}, \mathrm{NH}), 8.57(\mathrm{~s}, 1 \mathrm{H}, \mathrm{CH}=), 7.77(\mathrm{~d}, J=$ $\left.7.7 \mathrm{~Hz}, 1 \mathrm{H}, \mathrm{C}_{6} \mathrm{H}_{4} \mathrm{OCH}_{3}\right), 7.43(\mathrm{t}, J=7.8 \mathrm{~Hz}$, $\left.1 \mathrm{H}, \mathrm{C}_{6} \mathrm{H}_{4} \mathrm{OCH}_{3}\right), 7.32(\mathrm{dd}, J=8.4,5.7 \mathrm{~Hz}, 2 \mathrm{H}$, $\left.\mathrm{C}_{6} \mathrm{H}_{4} \mathrm{~F}\right), 7.15$ (t, $\left.J=8.8 \mathrm{~Hz}, 2 \mathrm{H}, \mathrm{C}_{6} \mathrm{H}_{4} \mathrm{~F}\right), 7.09$ $\left(\mathrm{d}, J=8.4 \mathrm{~Hz}, 1 \mathrm{H}, \mathrm{C}_{6} \mathrm{H}_{4} \mathrm{OCH}_{3}\right), 6.99(\mathrm{t}, J=$ $\left.7.5 \mathrm{~Hz}, 1 \mathrm{H}, \mathrm{C}_{6} \mathrm{H}_{4} \mathrm{OCH}_{3}\right), 4.62(\mathrm{dd}, J=9.4$, $4.2 \mathrm{~Hz}, 1 \mathrm{H}, \mathrm{CH}), 3.83$ (s, 3H, $\left.\mathrm{CH}_{3}\right), 3.38$ (dd, $\left.J=14.2,4.3 \mathrm{~Hz}, 1 \mathrm{H}, \mathrm{CH}_{2}\right), 3.04(\mathrm{dd}, J=14.1$, $9.5 \mathrm{~Hz}, 1 \mathrm{H}, \mathrm{CH}_{2}$ ). Anal. Calcd. for $\mathrm{C}_{18} \mathrm{H}_{16} \mathrm{FN}_{3} \mathrm{O}_{2} \mathrm{~S}$ : C, 60.49; H, 4.51; N, 11.76 . Found: C, 60.54; H, 4.58; N, 11.62 .

5 - ( 4 - Fl и о r obe nzyl) - 2 - I ( 4 methoxybenzylidene)-hydrazono]-thiazolidin4-one (6f). Yield 88 \%, m.p. $226-227{ }^{\circ} \mathrm{C} .{ }^{1} \mathrm{H}$ NMR (400 MHz, [D6]DMSO): $\delta=11.94$ (s, $1 \mathrm{H}, \mathrm{NH}), 8.30(\mathrm{~s}, 1 \mathrm{H}, \mathrm{CH}=), 7.66(\mathrm{~d}, J=$ $\left.8.7 \mathrm{~Hz}, 2 \mathrm{H}, \mathrm{C}_{6} \mathrm{H}_{4} \mathrm{OCH}_{3}\right), 7.32(\mathrm{dd}, J=8.3$, $\left.5.7 \mathrm{~Hz}, 2 \mathrm{H}, \mathrm{C}_{6} \mathrm{H}_{4} \mathrm{~F}\right), 7.15(\mathrm{t}, J=8.8 \mathrm{~Hz}, 2 \mathrm{H}$, $\left.\mathrm{C}_{6} \mathrm{H}_{4} \mathrm{~F}\right), 6.99$ (d, J=8.7 Hz, 2H, $\mathrm{C}_{6} \mathrm{H}_{4} \mathrm{OCH}_{3}$ ), $4.61(\mathrm{dd}, J=9.4,4.3 \mathrm{~Hz}, 1 \mathrm{H}, \mathrm{CH}), 3.79$ (s, $3 \mathrm{H}), 3.38$ (d, $\left.J=14.2,4.2 \mathrm{~Hz}, 1 \mathrm{H}, \mathrm{CH}_{2}\right), 3.03$ (dd, $J=14.1,9.5 \mathrm{~Hz}, 1 \mathrm{H}, \mathrm{CH}_{2}$ ). Anal. Calcd. for $\mathrm{C}_{18} \mathrm{H}_{16} \mathrm{FN}_{3} \mathrm{O}_{2} \mathrm{~S}$ : C, 60.49; H, 4.51; N, 11.76. Found: C, 60.60; H, 4.42; N, 11.88 . 
5 - (4 - Chlorobenzyl) - 2 - I (3, 4 dimethoxybenzylidene)-hydrazonol-thiazolidin-4-one (6g). Yield 89 \%, m.p. 214-215 ${ }^{\circ} \mathrm{C}$. ${ }^{1} \mathrm{H}$ NMR (400 MHz, [D6]DMSO): $\delta=11.86$ (s, 1H, NH), $8.27(\mathrm{~s}, 1 \mathrm{H}, \mathrm{CH}), 7.38(\mathrm{~d}, J=$ $\left.8.3 \mathrm{~Hz}, 2 \mathrm{H}, \mathrm{C}_{6} \mathrm{H}_{4} \mathrm{Cl}\right), 7.33-7.25(\mathrm{~m}, 4 \mathrm{H}$, $\left.\mathrm{C}_{6} \mathrm{H}_{3}\left(\mathrm{OCH}_{3}\right)_{2}+\mathrm{C}_{6} \mathrm{H}_{4} \mathrm{Cl}\right), 7.02(\mathrm{~d}, J=8.2 \mathrm{~Hz}$, $\left.1 \mathrm{H}, \mathrm{C}_{6} \mathrm{H}_{3}\left(\mathrm{OCH}_{3}\right)_{2}\right), 4.61(\mathrm{dd}, J=8.9,4.2 \mathrm{~Hz}$, $1 \mathrm{H}, \mathrm{CH}), 3.80\left(\mathrm{~s}, 3 \mathrm{H}, \mathrm{CH}_{3}\right), 3.77\left(\mathrm{~s}, 3 \mathrm{H}, \mathrm{CH}_{3}\right)$, 3.35 (dd, $J=14.2,4.3 \mathrm{~Hz}, 1 \mathrm{H}, \mathrm{CH}_{2}$ ), 3.07 (dd, $\left.J=14.2,8.9 \mathrm{~Hz}, 1 \mathrm{H}, \mathrm{CH}_{2}\right)$. Anal. Calcd. for $\mathrm{C}_{19} \mathrm{H}_{18} \mathrm{ClN}_{3} \mathrm{O}_{3} \mathrm{~S}: \mathrm{C}, 56.50 ; \mathrm{H}, 4.49 ; \mathrm{N}, 10.40$. Found: C, 56.55; H, 4.36; N, 10.46 .

2-(Benzylidenehydrazono) - 5-(4bromobenzyl)-thiazolidin-4-one (6h). Yield $82 \%$, m.p. $244-245^{\circ} \mathrm{C} .{ }^{1} \mathrm{H}$ NMR $(400 \mathrm{MHz}$, [D6]DMSO): $\delta=12.02(\mathrm{~s}, 1 \mathrm{H}, \mathrm{NH}), 8.37$ (s, $1 \mathrm{H}, \mathrm{CH}), 7.72(\mathrm{~d}, J=4.6 \mathrm{~Hz}, 2 \mathrm{H}, \mathrm{Ph}), 7.52$ $\left(\mathrm{d}, J=7.8 \mathrm{~Hz}, 2 \mathrm{H}, \mathrm{C}_{6} \mathrm{H}_{4} \mathrm{Br}\right), 7.44(\mathrm{~s}, 3 \mathrm{H}, \mathrm{Ph})$, 7.25 (d, $\left.J=7.4 \mathrm{~Hz}, 2 \mathrm{H}, \mathrm{C}_{6} \mathrm{H}_{4} \mathrm{Br}\right), 4.65$ (dd, $J=$ 9.2, 4.1 Hz, $1 \mathrm{H}, \mathrm{CH}), 3.37(\mathrm{~d}, 14.2, J=4.3 \mathrm{~Hz}$, $\left.1 \mathrm{H}, \mathrm{CH}_{2}\right), 3.04(\mathrm{dd}, J=13.7,9.7 \mathrm{~Hz}, 1 \mathrm{H}$, $\mathrm{CH}_{2}$ ). Anal. Calcd. for $\mathrm{C}_{17} \mathrm{H}_{14} \mathrm{BrN}_{3} \mathrm{OS}$ : C, 52.59; H, 3.63; N, 10.82. Found: C, 52.43; H, $3.66 ; \mathrm{N}, 10.88$.

2-[(4-Methoxybenzylidene)-hydrazono]-5(3-trifluoromethylbenzyl)-thiazolidin-4-one (6i). Yield $73 \%$, m.p. $218-219{ }^{\circ} \mathrm{C} .{ }^{1} \mathrm{H}$ NMR (400 MHz, [D6]DMSO): $\delta=11.97(\mathrm{~s}, 1 \mathrm{H}$, $\mathrm{NH}), 8.30(\mathrm{~s}, 1 \mathrm{H}, \mathrm{CH}=), 7.69-7.50(\mathrm{~m}, 6 \mathrm{H}$, $\left.\mathrm{Ar}, \mathrm{C}_{6} \mathrm{H}_{4} \mathrm{OCH}_{3}+\mathrm{C}_{6} \mathrm{H}_{4} \mathrm{CF}_{3}\right), 6.99(\mathrm{~d}, J=8.7 \mathrm{~Hz}$, $\left.2 \mathrm{H}, \mathrm{C}_{6} \mathrm{H}_{4} \mathrm{OCH}_{3}\right), 4.72(\mathrm{dd}, J=9.1,4.5 \mathrm{~Hz}, 1 \mathrm{H}$, $\mathrm{CH}), 3.79\left(\mathrm{~s}, 4 \mathrm{H}, \mathrm{CH}_{3}\right), 3.47(\mathrm{dd}, J=14.2$, $\left.4.5 \mathrm{~Hz}, 1 \mathrm{H}, \mathrm{CH}_{2}\right), 3.18(\mathrm{dd}, J=14.1,9.3 \mathrm{~Hz}$, $1 \mathrm{H}, \mathrm{CH}_{2}$ ). ). Anal. Calcd. for $\mathrm{C}_{19} \mathrm{H}_{16} \mathrm{~F}_{3} \mathrm{~N}_{3} \mathrm{O}_{2} \mathrm{~S}$ : C, 56.01; H, 3.96; N, 10.31. Found: C, 56.41; $\mathrm{H}, 3.85 ; \mathrm{N}, 10.24$.

2-[(2-Chlorobenzylidene)-hydrazono]-5(4-ethoxybenzyl)-thiazolidin-4-one (6j). Yield
77 \%, m.p. $228-229^{\circ} \mathrm{C} .{ }^{1} \mathrm{H}$ NMR $(400 \mathrm{MHz}$, [D6]DMSO): $\delta=12.01(\mathrm{~s}, 1 \mathrm{H}, \mathrm{NH}), 8.61(\mathrm{~s}$, $1 \mathrm{H}, \mathrm{CH}), 7.92\left(\mathrm{~d}, J=7.7 \mathrm{~Hz}, 1 \mathrm{H}, \mathrm{C}_{6} \mathrm{H}_{4} \mathrm{Cl}\right)$, $7.52\left(\mathrm{~d}, J=7.8 \mathrm{~Hz}, 1 \mathrm{H}, \mathrm{C}_{6} \mathrm{H}_{4} \mathrm{Cl}\right), 7.47$ (t, $J=$ $\left.7.5 \mathrm{~Hz}, 1 \mathrm{H}, \mathrm{C}_{6} \mathrm{H}_{4} \mathrm{Cl}\right), 7.41(\mathrm{t}, J=7.4 \mathrm{~Hz}, 1 \mathrm{H}$, $\left.\mathrm{C}_{6} \mathrm{H}_{4} \mathrm{Cl}\right), 7.18\left(\mathrm{~d}, J=8.3 \mathrm{~Hz}, 2 \mathrm{H}, \mathrm{C}_{6} \mathrm{H}_{4} \mathrm{OC}_{2} \mathrm{H}_{5}\right.$ ), $6.86\left(\mathrm{~d}, J=8.3 \mathrm{~Hz}, 2 \mathrm{H}, \mathrm{C}_{6} \mathrm{H}_{4} \mathrm{OC}_{2} \mathrm{H}_{5}\right), 4.60(\mathrm{~d}$, $J=9.3 \mathrm{~Hz}, 1 \mathrm{H}, \mathrm{CH}), 4.00(\mathrm{q}, J=6.7 \mathrm{~Hz}, 2 \mathrm{H}$, $\left.\mathrm{CH}_{3} \mathrm{CH}_{2} \mathrm{O}\right), 3.32(\mathrm{dd}, J=14.5,4.0 \mathrm{~Hz}, 1 \mathrm{H}$, $\mathrm{CH}_{2}$ ), 2.97 (dd, $J=14.0,9.6 \mathrm{~Hz}, 1 \mathrm{H}, \mathrm{CH}_{2}$ ), $1.31\left(\mathrm{t}, J=6.9 \mathrm{~Hz}, 3 \mathrm{H}, \mathrm{CH}_{3} \mathrm{CH}_{2} \mathrm{O}\right)$. Anal. Calcd. for $\mathrm{C}_{19} \mathrm{H}_{18} \mathrm{ClN}_{3} \mathrm{O}_{2} \mathrm{~S}$ : C, 58.83; H, 4.68; N, 10.83. Found: C, 58.88; H, 4.56; N, 10.74 .

Ethyl 4-\{2-[(3,4-dimethoxybenzylidene)hydrazono]-4-oxo-thiazolidin-5-ylmethyl? benzoate (6k). Yield $86 \%$, m.p. $215-216^{\circ} \mathrm{C}$. ${ }^{1} \mathrm{H}$ NMR (400 MHz, [D6]DMSO): $\delta=11.96$ $(\mathrm{s}, 1 \mathrm{H}, \mathrm{NH}), 8.27(\mathrm{~s}, 1 \mathrm{H}, \mathrm{CH}=), 7.91(\mathrm{~d}, J=$ $\left.8.1 \mathrm{~Hz}, 2 \mathrm{H}, \mathrm{C}_{6} \mathrm{H}_{5} \mathrm{COOC}_{2} \mathrm{H}_{5}\right), 7.43(\mathrm{~d}, J=$ $\left.8.1 \mathrm{~Hz}, 2 \mathrm{H}, \mathrm{C}_{6} \mathrm{H}_{5} \mathrm{COOC}_{2} \mathrm{H}_{5}\right), 7.28-7.26(\mathrm{~d}, J=$ $\left.8.2 \mathrm{~Hz}, 2 \mathrm{H}, \mathrm{C}_{6} \mathrm{H}_{3}\left(\mathrm{OCH}_{3}\right)_{2}\right), 7.01(\mathrm{~d}, J=8.1 \mathrm{~Hz}$, $\left.1 \mathrm{H}, \mathrm{C}_{6} \mathrm{H}_{3}\left(\mathrm{OCH}_{3}\right)_{2}\right), 4.67(\mathrm{dd}, J=9.0,4.5 \mathrm{~Hz}$, $1 \mathrm{H}, \mathrm{CH}), 4.30\left(\mathrm{q}, J=7.1 \mathrm{~Hz}, 2 \mathrm{H}, \mathrm{CH}_{3} \mathrm{CH}_{2} \mathrm{O}\right)$, $3.79\left(\mathrm{~s}, 3 \mathrm{H}, \mathrm{CH}_{3} \mathrm{O}\right), 3.76\left(\mathrm{~s}, 3 \mathrm{H}, \mathrm{CH}_{3} \mathrm{O}\right), 3.44$ (dd, $\left.J=14.1,4.3 \mathrm{~Hz}, 1 \mathrm{H}, \mathrm{CH}_{2}\right), 3.16$ (dd, $J=$ 14.1, $\left.9.2 \mathrm{~Hz}, 1 \mathrm{H}, \mathrm{CH}_{2}\right), 1.31(\mathrm{t}, J=7.1 \mathrm{~Hz}$, $3 \mathrm{H}, \mathrm{CH}_{3} \mathrm{CH}_{2} \mathrm{O}$ ). Anal. Calcd. for $\mathrm{C}_{22} \mathrm{H}_{23} \mathrm{~N}_{3} \mathrm{O}_{5} \mathrm{~S}$ : C, 59.85; H, 5.25; N, 9.52. Found: C, 59.48; $\mathrm{H}, 5.33 ; \mathrm{N}, 9.60$.

2-(Furan-2-ylmethylenehydrazono)-5-(3methoxybenzyl)-thiazolidin-4-one (7a). Yield 74 \%, m.p. $192-193{ }^{\circ} \mathrm{C} .{ }^{1} \mathrm{H}$ NMR $(400 \mathrm{MHz}$, [D6]DMSO): $\delta=12.00(\mathrm{~s}, 1 \mathrm{H}, \mathrm{NH}), 8.19(\mathrm{~s}$, $1 \mathrm{H}, \mathrm{CH}=), 7.84\left(\mathrm{~s}, 1 \mathrm{H}, \mathrm{C}_{6} \mathrm{H}_{4} \mathrm{OCH}_{3}\right), 7.24(\mathrm{t}$, $\left.\mathrm{J}=7.8 \mathrm{~Hz}, 1 \mathrm{H}, \mathrm{C}_{6} \mathrm{H}_{4} \mathrm{OCH}_{3}\right), 6.94(\mathrm{~d}, \mathrm{~J}=3.3$ $\left.\mathrm{Hz}, 1 \mathrm{H}, \mathrm{C}_{4} \mathrm{H}_{3} \mathrm{O}\right)$, 6.87-6.80 (m, $3 \mathrm{H} \mathrm{C}_{4} \mathrm{H}_{3} \mathrm{O}+$ $\left.\mathrm{C}_{6} \mathrm{H}_{4} \mathrm{OCH}_{3}\right), 6.65-6.61\left(\mathrm{~m}, 1 \mathrm{H}, \mathrm{C}_{4} \mathrm{H}_{3} \mathrm{O}\right), 4.64$ $(\mathrm{dd}, \mathrm{J}=9.9,4.2 \mathrm{~Hz}, 1 \mathrm{H}, \mathrm{CH}), 3.38(\mathrm{~d}, \mathrm{~J}=14.2$, $\left.4.2 \mathrm{~Hz}, 1 \mathrm{H}, \mathrm{CH}_{2}\right), 2.96(\mathrm{dd}, \mathrm{J}=13.9,10.0 \mathrm{~Hz}$, 
$1 \mathrm{H}, \mathrm{CH}_{2}$ ). Anal. Calcd. for $\mathrm{C}_{16} \mathrm{H}_{15} \mathrm{~N}_{3} \mathrm{O}_{3} \mathrm{~S}: \mathrm{C}$, 58.35; H, 4.59; N, 12.76. Found: C, 58.52; H, $4.54 ; \mathrm{N}, 12.55$.

2-(Furan-2-ylmethylenehydrazono)-5-(3trifluoromethyl-benzyl)-thiazolidin-4-one (7b). Yield 80 \%, m.p. 195-196 ${ }^{\circ} \mathrm{C} .{ }^{1} \mathrm{H}$ NMR (400 MHz, [D6]DMSO): $\delta=12.03(\mathrm{~s}, 1 \mathrm{H}$, $\mathrm{NH}), 8.19(\mathrm{~s}, 1 \mathrm{H}, \mathrm{CH}=), 7.85\left(\mathrm{~s}, 1 \mathrm{H}, \mathrm{C}_{6} \mathrm{H}_{4} \mathrm{CF}_{3}\right)$, 7.67-7.54 (m, 4H, $\left.\mathrm{C}_{4} \mathrm{H}_{3} \mathrm{O}+\mathrm{C}_{6} \mathrm{H}_{4} \mathrm{OCH}_{3}\right), 6.94$ $\left(\mathrm{d}, J=3.4 \mathrm{~Hz}, 1 \mathrm{H}, \mathrm{C}_{4} \mathrm{H}_{3} \mathrm{O}\right), 6.63(\mathrm{dd}, J=3.3$, $1.7 \mathrm{~Hz}, 1 \mathrm{H}, \mathrm{C}_{4} \mathrm{H}_{3} \mathrm{O}$ ) , 4.73 (dd, $J=9.1,4.6 \mathrm{~Hz}$, $1 \mathrm{H}, \mathrm{CH}), 3.46$ (dd, $J=14.1,4.5 \mathrm{~Hz}, 1 \mathrm{H}, \mathrm{CH}_{2}$ ), $3.17\left(\mathrm{dd}, J=14.1,9.2 \mathrm{~Hz}, 1 \mathrm{H}, \mathrm{CH}_{2}\right)$. Anal. Calcd. for $\mathrm{C}_{16} \mathrm{H}_{12} \mathrm{~F}_{3} \mathrm{~N}_{3} \mathrm{O}_{2} \mathrm{~S}$ : C, 52.31; H, 3.29; N, 11.44. Found: C, 52.44; H, 3.25; N, 11.48.

Antibacterial data collection. Inhibition of bacterial growth was determined measuring absorbance at $600 \mathrm{~nm}$ (OD600), using a Tecan M1000 Pro monochromator plate reader. The percentage of growth inhibition was calculated for each well, using the negative control (media only) and positive control (bacteria without inhibitors) on the same plate as references.

Antifungal data collection. Growth inhibition of $C$. albicans was determined measuring absorbance at $530 \mathrm{~nm}$ (OD530), while the growth inhibition of $C$. neoformans was determined measuring the difference in absorbance between 600 and $570 \mathrm{~nm}$ (OD600-570), after the addition of resazurin $(0.001 \%$ final concentration) and incubation at $35^{\circ} \mathrm{C}$ for additional $2 \mathrm{~h}$. The absorbance was measured using a Biotek Synergy HTX plate reader. The percentage of growth inhibition was calculated for each well, using the negative control (media only) and positive control (bacteria without inhibitors) on the same plate as references.
Inhibition. Percentage growth inhibition of an individual sample is calculated based on Negative controls (media only) and Positive Controls (bacterial/fungal media without inhibitors). Negative inhibition values indicate that the growth rate (or OD600) is higher compared to the Negative Control (Bacteria/fungi only, set to $0 \%$ inhibition). The growth rate for all bacteria and fungi has a variation of $-/+$ $10 \%$, which is within the reported normal distribution of bacterial/fungal growth.

\section{Results and Discussion}

Previously, we have developed $[8,9]$ a convenient preparative method for the synthesis of 2-substituted 5-benzyl-4-thiazolidinones via cyclization of ethyl-(2-bromo-3-aryl)-propanoates with $\mathrm{S}, \mathrm{N}$-nucleophiles. Using this approach, we obtained a series of new 5-R-benzyl2-(arylidenehydrazono)thiazolidin-4-one.

Arendiazonium bromides react with ethyl acrylate in the Meerwein reaction condition [17] to form ethyl 2-bromo-3-arylpropanoates 3a-j. These esters were converted into target 5-R-benzyl-2-(arylidenehydrazono)thiazolidin4-one $6 \mathbf{a - k}$ and $7 \mathbf{a}, \mathbf{b}$ via reaction with thiosemicarbazones $\mathbf{4 a - d}$ and $\mathbf{5}$ according to the previously reported synthetic protocols [18] (scheme).

Antimicrobial activity. The antimicrobial screening was performed by CO-ADD (the Community for Antimicrobial Drug Discovery) funded by the Wellcome Trust (UK) and the University of Queensland (Australia) [19]. The growth inhibition was measured against five bacterial strains (Escherichia coli, Klebsiella pneumoniae, Acinetobacter baumannii, Pseudomonas aeruginosa and Staphylococcus aureus) and two fungal strains (Candida albi- 
1a-j, 3a-j: $\mathrm{R}=\mathrm{H}(\mathbf{a}) ; 2-\mathrm{CH}_{3}(\mathbf{b})$;

$3-\mathrm{CH}_{3}(\mathbf{c}) ; 4-\mathrm{C}_{2} \mathrm{H}_{5}(\mathbf{d})$;

4-F(e); 4-Cl(f); 4-Br(g);

$3-\mathrm{CF}_{3}(\mathbf{h}) ; 4-\mathrm{C}_{2} \mathrm{H}_{5}(\mathbf{i})$;

$4-\mathrm{COOC}_{2} \mathrm{H}_{5}(\mathrm{j})$.
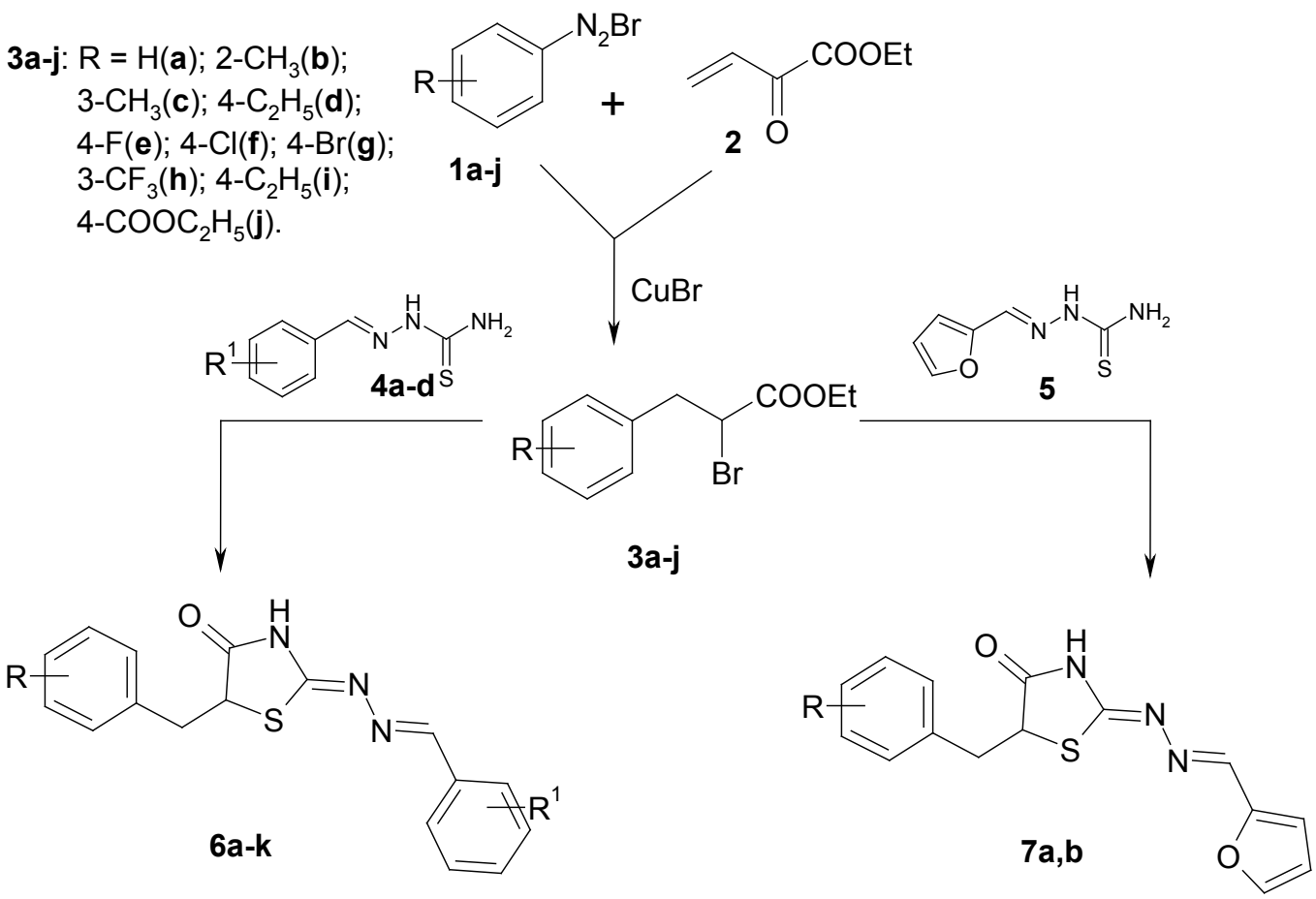

4a-d: $\mathrm{R}=\mathrm{H}(\mathbf{a}) ; 2-\mathrm{CH}_{3} \mathrm{O}(\mathbf{b}) ; 4-\mathrm{CH}_{3} \mathrm{O}(\mathbf{c}) ; 3,4-\left(\mathrm{CH}_{3}\right)_{2}(\mathbf{d}$

6a-k: $\mathrm{R}=\mathrm{H}, \mathrm{R}^{1}=2-\mathrm{CH}_{3} \mathrm{O}(\mathbf{a}) ; \mathrm{R}=2-\mathrm{CH}_{3}, \mathrm{R}^{1}=\mathrm{H}(\mathbf{b}) ; \mathrm{R}=4-\mathrm{C}_{2} \mathrm{H}_{5}, \mathrm{R}^{1}=4-\mathrm{CH}_{3} \mathrm{O}(\mathbf{c})$;

$\mathrm{R}=4-\mathrm{F}, \mathrm{R}^{1}=\mathrm{H}(\mathbf{d}) ; \mathrm{R}=4-\mathrm{F}, \mathrm{R}^{1}=2-\mathrm{CH}_{3} \mathrm{O}(\mathbf{e}) ; \mathrm{R}=4-\mathrm{F}, \mathrm{R}^{1}=4-\mathrm{CH}_{3} \mathrm{O}(\mathbf{f})$;

$\mathrm{R}=4-\mathrm{Cl}, \mathrm{R}^{1}=3,4-\left(\mathrm{CH}_{3} \mathrm{O}\right)_{2}(\mathbf{g}) ; \mathrm{R}=4-\mathrm{Br}, \mathrm{R}^{1}=\mathrm{H}(\mathbf{h}) ; \mathrm{R}=3-\mathrm{CF}_{3}, \mathrm{R}^{1}=4-\mathrm{CH}_{3} \mathrm{O}(\mathbf{i})$;

$\mathrm{R}=4-\mathrm{C}_{2} \mathrm{H}_{5} \mathrm{O}, \mathrm{R}^{1}=2-\mathrm{Cl}(\mathbf{j}) ; \mathrm{R}=4-\mathrm{COOC}_{2} \mathrm{H}_{5}, \mathrm{R}^{1}=3,4-\left(\mathrm{CH}_{3} \mathrm{O}\right)_{2}(\mathbf{k})$.

7a,b: $\mathrm{R}=3-\mathrm{CH}_{3} \mathrm{O}(\mathbf{a}) ; 3-\mathrm{CF}_{3}(\mathbf{b})$.

Scheme. Synthesis of 5-R-benzyl-2-(arylidenehydrazono)thiazolidin-4-ones.

cans and Cryptococcus neoformans). The standard concentration employed for screening was $32 \mathrm{mg} / \mathrm{mL}$ in DMSO. The observed in vitro antimicrobial activities of our synthesized products $6 \mathbf{a - k}$ and $7 \mathbf{a}, \mathbf{b}$ are presented in Table.

According to the results of antimicrobial screening, the majority of the tested compounds exhibit [a] moderate antibacterial activity against Staphylococcus aureus and
Acinetobacter baumannii strains and antifungal activity against Cryptococcus neoformans. The appearance of antistaphylococcal activity of the studied substances is facilitated by both unsubstituted 2-benzylidene hydrazone fragments $(\mathbf{6 b}, \mathbf{h}, \mathrm{GI}=17.6 \%$ and $21.9 \%$, respectively) and 2-chloro- $(\mathbf{6 j}$, GI $=22.0 \%)$, 4-methoxy- (6i, GI $=19.6 \%)$ and especially 3,4-dimethoxy $(6 \mathbf{k}, \mathrm{GI}=33.3 \%)$ substituents. 
Table. Antimicrobial activity of compounds $6 a-k$ and $7 \mathbf{a}, \mathbf{b}$.

\begin{tabular}{|c|c|c|c|c|c|c|c|}
\hline $\begin{array}{l}\bar{\Xi} \\
\overline{0} \\
\stackrel{0}{0} \\
ن\end{array}$ & 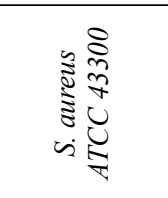 & 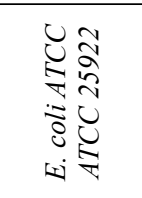 & 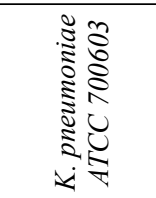 & 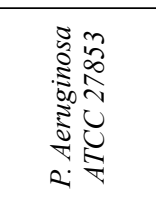 & 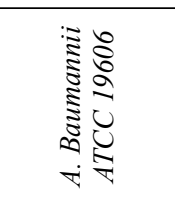 & 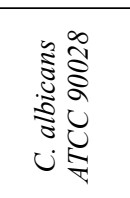 & 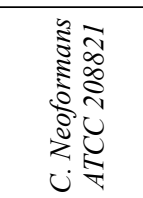 \\
\hline $6 a$ & $-4.8 ;-6.4$ & $3.3 ; 4.3$ & $-6.9 ; 10.9$ & $3.3 ; 4.4$ & $-8.3 ;-9.0$ & $5.8 ; 6.5$ & $18.4 ; 21.8$ \\
\hline $6 \mathrm{~b}$ & $13.7 ; 17.6$ & $-2.1 ; 4.1$ & $1.8 ; 6.0$ & $-1.2 ;-1.7$ & $-2.6 ;-6.0$ & $-1.9 ; 9.7$ & $-5.3 ; 0.0$ \\
\hline $6 c$ & $-10.4 ;-6.0$ & $0.3 ; 2.2$ & $-3.6 ;-5.8$ & $3.4 ; 7.1$ & $-8.4 ; 13.6$ & $1.8 ; 7.8$ & $13.6 ; 19.5$ \\
\hline $6 d$ & $-6.1 ; 1.3$ & $3.1 ; 9.8$ & $-19.1 ; 7.9$ & $12.4 ; 12.6$ & $-21.0 ; 4.9$ & $2.1 ; 3.7$ & $25.6 ; 28.6$ \\
\hline $6 e$ & $11.5 ; 14.8$ & $-0.8 ; 6.2$ & $5.8 ; 6.2$ & $0.1 ; 4.7$ & $-0.0 ;-4.4$ & $-1.2 ; 4.7$ & $0.8 ; 7.0$ \\
\hline $6 f$ & $5.5 ; 7.8$ & $4.1 ; 5.4$ & $-11.1 ; 4.7$ & $5.5 ; 7.6$ & $-14.1 ;-18.1$ & $3.8 ; 4.5$ & $16.1 ; 21.1$ \\
\hline $6 g$ & $11.0 ; 14.3$ & $5.3 ; 7.3$ & $-2.3 ; 10.1$ & $4.4 ; 5.8$ & $1.2 ; 6.5$ & $3.2 ; 5.0$ & $23.2 ; 25.2$ \\
\hline $6 \mathrm{~h}$ & $15.4 ; 21.9$ & $-4.8 ; 0.3$ & $-14.8 ;-2.5$ & $-1.7 ; 0.1$ & $-1.0 ; 3.5$ & $2.2 ; 2.3$ & $16.1 ; 18.0$ \\
\hline $6 \mathbf{i}$ & $15.8 ; 19.6$ & $-1.9 ; 8.2$ & $7.2 ; 8.0$ & $-2.6 ; 1.0$ & $15.3 ; 4.2$ & $1.6 ; 5.4$ & $-3.4 ;-4.6$ \\
\hline $6 \mathbf{j}$ & $15.2 ; 22.0$ & $3.8 ; 7.4$ & $10.2 ; 4.0$ & $3.6 ; 5.2$ & $-6.2 ;-8.2$ & $1.2 ; 5.3$ & $12.9 ; 16.4$ \\
\hline $6 k$ & $19.6 ; 33.3$ & $-1.3 ; 7.3$ & $5.6 ; 7.6$ & $5.3 ; 5.8$ & $14.1 ; 6.0$ & $1.2 ; 5.2$ & $1.3 ; 6.2$ \\
\hline $7 a$ & $18.8 ; 23.1$ & $-1.1 ;-4.7$ & $-0.1 ; 0.5$ & $3.7 ; 6.7$ & $-1.0 ;-1.3$ & $2.3 ; 2.9$ & $-0.8 ;-4.7$ \\
\hline $7 b$ & $-2.3 ;-6.6$ & $4.2 ; 5.6$ & $4.4 ; 6.3$ & $10.5 ; 7.0$ & $16.4 ; 17.7$ & $4.8 ; 8.1$ & $20.0 ; 19.4$ \\
\hline
\end{tabular}

Additionally, oxygen-containing 2-furfurylidene derivative $7 \mathbf{a}$ also shows the antistaphylococcal activity $(\mathrm{GI}=23.1 \%)$.

The Activity against Cryptococcus neoformans (GI in the range of $19.5-23.2 \%$ ) was observed in compounds that also contain the methoxy groups in the arylidenehydrazone moiety, combined with both unsubstituted benzyl group (6a) and para-ethyl- (6c), parafluoro- (6f) and para-chloro- (6g) substituents, and unsubstituted 2-benzylidenehydrazone4-thiazolidinones combined in a molecule with 5-para-fluoro- (6d) and para-bromo- (6h) benzyl substituents $(\mathrm{GI}=28.6 \%$ and $18.0 \%$, respectively).

\section{Conclusions}

A series of new 5-R-benzyl-2-(arylidenehydrazono)thiazolidin-4-ones were prepared. The antimicrobial screening of them against Gram positive, Gram negative microorganisms and fungi was performed and the results are described in this paper. It was found that some derivatives are active against Staphylococcus aureus and Cryptococcus neoformans.

\section{Acknowledgements}

We are grateful to the Community for Antimicrobial Drug Discovery (funded by the Wellcome Trust (UK) and the University of Queensland (Australia), for evaluation of antimicrobial activity.

This work was financially supported by the National Research Foundation of Ukraine (project 2020.01/0166).

\section{REFERENCES}

1. Tomasić T, Masic L. Rhodanine as a privileged scaffold in drug discovery. Curr Med Chem. 2009; 16(13): 1596-629.

2. Kaminskyy D, Kryshchyshyn A, Lesyk R. Recent developments with rhodanine as a scaffold for drug 
discovery. Expert Opin Drug Discov. 2017; 12(12): 1233-52.

3. Kaminskyy D, Kryshchyshyn A, Lesyk R. 5-Ene4-thiazolidinones - An efficient tool in medicinal chemistry. Eur J Med Chem. 2017; 140: 542-94.

4. Drapak I, Foliush V, Chaban T, Matiychuk V. Synthesis, antimicrobial and antitumor activities of 2-[5-(2-R-benzyl)thiazol-2-ylimino]thiazolidin4-ones. Biointerface Res Appl Chem. 2020; 10(3): 5507-11.

5. De Monte C, Carradori S, Bizzarri B, Bolasco A, Caprara F, Mollica A, Rivanera D, Mari E, Zicari A, Akdemir A, Secci D. Anti-Candida activity and cytotoxicity of a large library of new N-substituted-1,3- thiazolidin-4-one derivatives. Eur J Med Chem. 2016; 107: 82-96.

6. El-Sayed WA, Abdel-Monem YK, Yousif NM, Tawfek N, Shaaban MT, Abdel-Rahman AA. Antimicrobial activity of new 2,4-disubstituted thiazolidinone derivatives. Z Naturforsch C J Biosci. 2009; 64(1112): 785-9.

7. Oniga O, Ndongo JT, Cristina N, Tiperciuc B, Oniga S, Pîrnău A, Vlase L, Verité P. Synthesis and antimicrobial activity of some new 2-hydrazonethiazoline-4- ones. Farmacia. 2012; 60(6): 785-97.

8. Belwal CK, Rajyaguru CM, Joshi KA. Synthesis and antimicrobial screening of 2-[(5,6-dimethoxy-2,3dihydro-1H-inden-1-ylidene)hydrazinylidine]-1,3thiazolidin-4-one and its 5-Arylidine derivatives. Heterocycl Lett. 2014; 4(1): 65-71.

9. Hassan A, Shaaban M, Nasr MK, El-Shaieb K, Abdel-Aziz M, Abdel-Aziz A. Synthesis and Antibacterial Activity of New Substituted Ethylidenehydrazinylidene-1,3-thiazol-4-ones. J Chem Res. 2014; 38(11): $673-8$.

10. Goudreau N, Hucke O, Faucher AM, Grand-Mấtre C, Lepage O, Bonneau PR, Mason SW, Titolo $S$. Discovery and Structural Characterization of a New Inhibitor Series of HIV-1 Nucleocapsid Function: NMR Solution Structure Determination of a Ternary Complex Involving a 2:1 Inhibitor/NC Stoichiometry. J Mol Biol. 2013; 425(11): 1982-98.

11. Singh IP, Saxena AK, Sinha JN, Shanker K. Synthesis and anti-inflammatory activity of thiazolidinones and imidazolidinones derived from thiosemicarbazones. Eur J Med Chem. 1985; 20(3): 283-6.

12. Abbas EMH, Gomha SM, Farghaly TA, Abdalla MM. Synthesis of New Thiazole Derivatives as Antitumor Agents. Curr Org Synth. 2016; 13(3): 456-65.

13. El-Mekabaty A, Habib OMO, El-Moneim MA, Hussein $A S$. Efficient and convenient route to the synthesis of some novel sulfonate ester-based heterocycles as antitumor agents. Heterocycles. 2018; 96(4): 677-89.

14. Khan A, Diwan A, Thabet HK, Imran M, Bakht MA. Discovery of Novel Pyridazine-Based Cyclooxygenase-2 Inhibitors with a Promising Gastric Safety Profile. Molecules. 2020; 25(9): 20022.

15. Khan A, Diwan A, Thabet HK, Imran M. Synthesis of novel N-substitutedphenyl-6-oxo-3-phenylpyridazine derivatives as cyclooxygenase- 2 inhibitors. Drug Dev Res. 2020; 81(5): 573-84.

16. Channar PA, Saeed A, Larik FA, Rafiq M, Ashraf Z, Jabeen F, Fattah TA. Synthesis, computational studies and enzyme inhibitory kinetics of substituted methyl [2-((4-dimethylamino-benzylidene)hydrazono)-4-oxo-thiazolidin-5-ylidene]acetates as mushroom tyrosinase inhibitors. Bioorg Med Chem. 2017; 25(21): 5929-38.

17. Obushak ND, Lesyuk AI, Gorak YI, Matiichuk VS. Mechanism of Meerwein arylation of furan derivatives. Rus J Org Chem. 2009; 45(9):1375-81, doi: 10.1134/S1070428009090103.

18. Obushak ND, Matiichuk VS, Ganushchak NI, Burlak $Y u E$. Synthesis of heterocycles from products of the anion arylation of unsaturated compounds. 3 . 2-Arylimino-5-arylmethyl-4-thiazolidones. Chem Heterocycl Compd. 1998; 34(4): 492-6.

19. Open-access antimicrobial screening program: https://www.co-add.org

\section{Синтез та антимікробна активність 5-R-бензил- 2-(ариліденгідразоно)тіазолідин-4-онів}

I. В. Драпак, Л. С. Логойда, Н. Є. Штойко, М. I. Сулима, Т. I. Чабан, В. С. Матійчук

Мета. Синтез та дослідження антимікробної активності 5-R-бензил-2-(ариліденгідразоно)тіазолідин-4онів. Методи. Органічний синтез, аналітичні та спек- 
тральні методи, антимікробна активність. Результати. 5-R-бензил-2-(ариліденгідразоно)тіазолідин-4-они 6a-k i $\mathbf{7 a}, \mathbf{b}$ отримували 3 хорошими виходами реакцією 2-бромо-3-арилпропаноатів 3a-j 3 тіосемікарбазонами ароматичних альдегідів і фурфуролу. Їх структури були підтверджені ${ }^{1} \mathrm{H}$ ЯМР спектроскопією та даними елементного аналізу. Синтезовані сполуки досліджували на антимікробну активність щодо п'яти бактеріальних штамів (Escherichia coli, Klebsiella pneumoniae, Acinetobacter baumannii, Pseudomonas aeruginosa, Staphylococcus aureus) та двох грибкових штамів (Candida albicans, Cryptococcus neoformans). Результати антимікробної активності свідчать про те, що сполуки 6b, 6h-k та 7a виявляють помірну активність щодо грам-позитивного бактеріального штаму Staphylococcus aureus, сполуки 6c, 6f, 6i, 6k та 7b - щодо грамнегативного бактеріального штаму Acinetobacter baumannii, а сполуки 6a, 6c, 6d, 6f, 6g, 6h та $7 \mathbf{b}$ - щодо грибкового штаму Cryptococcus neoformans. Висновки. Отримано ряд нових 5-R-бензил-2-(ариліденгідразоно) тіазолідин-4-онів. Встановлено, що переважна більшість синтезованих сполук виявляє помірну антимікробну активність.

К л ю ч о в і с л о в а: органічний синтез, арилювання, тіазолідин-4-они, антимікробна активність.

\section{Синтез и антимикробная активность 5-R-бензил-2 (арилиденгидразоно) тиазолидин- 4-онов}

И. В. Драпак, Л. С. Логойда, Н. Е. Штойко, М. И. Сулима, Т. И. Чабан, В. С. Матийчук

Цель. Синтез и исследование антимикробной активности 5-R-бензил-2-(арилиденгидразоно)тиазоли- дин-4-онов. Методы. Органический синтез, аналитические и спектральные методы, антимикробная активность. Результаты. 5-R-бензил-2-(арилиденгидразоно)тиазолидин-4-оны 6a-k и 7a, b получали с хорошими выходами реакцией 2-бром-3-арилпропаноатов 3a-j с тиосемикарбазонамы ароматических альдегидов и фурфурола. Их структуры были подтверждены ${ }^{1} \mathrm{H}$ ЯМР спектроскопией и данными элементного анализа. Синтезированные соединения исследовали на антимикробную активность в отношении пяти бактериальных штаммов (Escherichia coli, Klebsiella pneumoniae, Acinetobacter baumannii, Pseudomonas aeruginosa, Staphylococcus aureus) и двух грибковых штаммов (Candida albicans, Cryptococcus neoformans). Результаты антимикробной активности свидетельствуют о том, что соединения 6b, 6h-k и 7a проявляют умеренную активность относительно грамм-положительного бактериального штамма Staphylococcus aureus, соединения $\mathbf{6 c}, \mathbf{6 f}, \mathbf{6 i}, \mathbf{6 k}$ и $7 \mathbf{b}-$ относительно грамм- отрицательного бактериального штамма Acinetobacter baumannii, а соединения 6а, $\mathbf{6 c}, \mathbf{6 d}, \mathbf{6 f}$, $\mathbf{6 g}, \mathbf{6 h}$ и $\mathbf{7 b}$ - относительно грибкового штамма Cryptococcus neoformans. Выводы. Получен ряд новых 5-R-бензил-2-(арилиденгидразоно)тиазолидин-4-онов. Установлено, что большинство синтезированных соединений проявляет умеренное антимикробное действие.

Кл юч е в ы е сл ов а: органический синтез, арилирование, 5-R-бензил-2-(арилиденгидразоно)тиазолидин-4-оны, антимикробная активность.

Received 04.09.2020 